\title{
Negativicoccus succinicivorans gen. nov., sp. nov., isolated from human clinical samples, emended description of the family Veillonellaceae and description of Negativicutes classis nov., Selenomonadales ord. nov. and Acidaminococcaceae fam. nov. in the bacterial phylum Firmicutes
}

\section{Correspondence \\ Hélène Marchandin \\ h-marchandin@chu-montpellier.fr}

\author{
Hélène Marchandin, ${ }^{1,2}$ Corinne Teyssier, ${ }^{1}$ Josiane Campos, ${ }^{2}$ \\ Hélène Jean-Pierre, ${ }^{1,2}$ Frédéric Roger, ${ }^{1}$ Bernard Gay, ${ }^{3}$ \\ Jean-Philippe Carlier ${ }^{4}$ and Estelle Jumas-Bilak ${ }^{1}$
}

\footnotetext{
1 Université Montpellier 1, EA 3755, Laboratoire de Bactériologie-Virologie, Faculté de Pharmacie, 15, Avenue Charles Flahault, BP 14491, 34060 Montpellier Cedex 5, France

${ }^{2}$ Centre Hospitalier et Universitaire de Montpellier, Hôpital Arnaud de Villeneuve, Laboratoire de Bactériologie, 371 Avenue du Doyen Gaston Giraud, 34295 Montpellier Cedex 5, France

${ }^{3}$ Université Montpellier 1 et Université Montpellier 2, UMR 5236-CPBS CNRS, Centre d'études d'agents pathogènes et Biotechnologies pour la Santé, Institut de Biologie, 4, Boulevard Henri IV, CS 69033, 34965 Montpellier Cedex 2, France

${ }^{4}$ Institut Pasteur, Centre National de Référence des Bactéries Anaérobies et du Botulisme, 25 rue du Dr Roux, 75724 Paris Cedex 15, France
}

Three strains of a hitherto unknown, Gram-negative, tiny, anaerobic coccus were collected from human clinical samples originating from skin and soft tissues. The three isolates displayed at least $99.9 \%$ identity in their $16 \mathrm{~S}$ rRNA gene sequences and more than $99.8 \%$ identity in their dnaK gene sequences. The isolates were affiliated to the family Veillonellaceae, the coccobacillus Dialister micraerophilus being the most closely related species, but there was no more than $91.1 \%$ identity in the 16S rRNA gene sequence between this species and the three isolates. Phylogeny based on the 16S rRNA gene confirmed that the three strains represent a novel and robust lineage within the current family Veillonellaceae. A similar genomic structure was demonstrated for the three isolates by PFGE-based analysis. Morphology and metabolic end products, as well as genotypic and phylogenetic data supported the proposal of the novel genus Negativicoccus gen. nov., with the novel species Negativicoccus succinicivorans sp. nov. [type strain ADV 07/08/06-B-1388 ${ }^{\top}$ (=AIP $149.07^{\top}=$ CIP $109806^{\top}=$ DSM $21255^{\top}=$ CCUG $56017^{\top}$ ) as type species]. Phylogenetic analyses based on the 16S rRNA gene sequences of members of the phylum Firmicutes and other phyla indicated that the family Veillonellaceae forms a robust lineage clearly separated from those of the classes 'Bacill', 'Clostridia', Thermolithobacteria and 'Erysipelotrichi' in the phylum Firmicutes. Therefore, we propose that this family is a class-level taxon in the phylum Firmicutes, for which the name Negativicutes classis nov. is proposed, based on the Gram-negative type of cell wall of its members, with the type order Selenomonadales ord. nov. In this order, a novel family,

Acidaminococcaceae fam. nov., is proposed and description of the family Veillonellaceae is emended.

The GenBank/EMBL/DDBJ accession numbers for the 16S rRNA gene and dnaK gene sequences of Negativicoccus succinicivorans gen. nov., sp. nov. ADV 07/08/06-B-1388' are FJ715930 and FJ715931, respectively.

PFGE migration of I-Ceul-restricted DNAs, an ML tree based on 227 partial 16S rRNA gene sequences (1200 nt), showing relationships between members of the family Veillonellaceae, other members of the phylum Firmicutes and other selected phyla, and composition of datasets A and B are available with the online version of this paper. 
Anaerobic, Gram-negative cocci are currently classified in four genera of the family Veillonellaceae (Garrity \& Holt, 2001): Veillonella, Acidaminococcus, Megasphaera and Anaeroglobus (Prévot, 1933; Rogosa, 1969, 1971; Carlier et al., 2002). These four genera can be distinguished by phenotypic and genotypic features, mainly metabolic end products, and $16 \mathrm{~S}$ rRNA and dnaK gene sequence analysis.

The family Veillonellaceae, formerly 'Acidaminococcaceae', is currently classified in the phylum Firmicutes (low-G +C Gram-positive bacteria), class Clostridia, one of the three classes currently recognized in the phylum together with 'Bacilli' and Thermolithobacteria (Sokolova et al., 2007), and in the order Clostridiales, which comprises 19 families (including nine families incertae sedis) (Ludwig et al., 2009). Despite the establishment of a revised road map to the phylum Firmicutes by Ludwig et al. (2009), significant further reorganization is expected in this taxon. For example, Ludwig et al. (2009) also suggested that the family Erysipelotrichaceae should be elevated to the rank of class, for which the name 'Erysipelotrichi' was proposed, but this has not yet been validly published. From a previous highlevel phylogenetic study, we proposed an emended description of the family Syntrophomonadaceae, order Clostridiales, and suggested that it should be elevated to a higher rank than that of family (Jumas-Bilak et al., 2009).

The aim of this study was to describe a novel genus and species in the family Veillonellaceae. The relationships among members of the current family Veillonellaceae with other members of the phylum Firmicutes were also explored in order to propose a reappraisal of their taxonomic level.

\section{Negativicoccus succinicivorans gen. nov., sp. nov., isolated from human clinical samples}

The three strains studied were isolated from three patients hospitalized at the University Hospital of Montpellier, France, between January 2004 and August 2006 (ADV 07/ 08/06-B- $1388^{\mathrm{T}}=$ AIP $149.07^{\mathrm{T}}$, ADV 12/01/04-B-1195=AIP 150.07 and ADV 03/08/05-B-3158=AIP 147.07). The three strains were isolated from skin or soft tissue samples. The isolates grew on Columbia sheep blood agar (bioMérieux) after 2 to 3 days incubation at $37{ }^{\circ} \mathrm{C}$ in an anaerobic jar with the Anaerogen System (Oxoid Unipath). All isolates were recovered in mixed aerobic/anaerobic flora. These isolates were further submitted to polyphasic investigation.

The 16S rRNA gene and $70 \mathrm{kDa}$ heat-shock protein gene (dnaK) were selectively amplified by PCR as described previously (Carlier et al., 2002; Marchandin et al., 2003b). The dnaK gene sequences were translated to the Hsp70 amino acid sequence using TRANSLATE (www.expasy.ch) to search for diderm insert signature sequences that distinguish Gram-negative bacteria from Gram-positive bacteria (Gupta, 1998). Using LALIGN software (www.expasy.org), the three isolates were shown to share at least $99.9 \%$ of their 16S rRNA gene sequence (GenBank accession numbers FJ715928-FJ715930) and at least $99.8 \%$ of their
dnaK gene sequences (FJ715931-FJ715933). Nearly complete $16 \mathrm{~S}$ rRNA gene sequences (1401 nt) and partial dnaK sequences (about $650 \mathrm{nt}$ ) were matched with GenBank and EMBL databases using the BLAST program (Altschul et al., 1997). They displayed a maximum level of identity with members of the genus Dialister within the family Veillonellaceae (Willems \& Collins, 1995; Garrity \& Holt, 2001), the most closely related known species being Dialister micraerophilus with sequence identity of about $91.1 \%$. The maximum $16 \mathrm{~S}$ rRNA gene sequence identity (more than $99.7 \%$ ) was observed with sequences of several uncultured bacterial clones from the human skin microbiome (GenBank accession number GQ014591, for example) (Grice et al., 2009) and with uncultured clone rRNA030 from human vaginal epithelium (GenBank accession number AY958803). The highest levels of dnaK gene sequence identity were observed with members of the genera Veillonella, Megasphaera and Dialister, but were no higher than $74.2-76.2 \%$. Hsp70 proteins lacked the diderm insert observed for Firmicutes, Actinobacteria and Archaea (Gupta, 1998). 16S rRNA gene-based phylogenetic analyses were conducted on sequences selected from the GenBank database to study the relationships between the unknown isolates and species of the current family Veillonellaceae. The sequences were aligned using CLC Sequence Viewer, version 5.11 (Knudsen et al., www.clcbio.com). The regions of uncertain alignment were visually and automatically determined using Gblocks 0.91b software (Castresana, 2000) and then manually removed using Sequence Alignment Editor software (Se-Al Version 2.0a11; http:// tree.bio.ed.ac.uk/software/seal/) before reconstruction of phylogenetic trees. Phylogenetic trees were inferred by using distance and maximum-likelihood methods. Neighbour-joining (NJ) and maximum-likelihood (ML) evolutionary trees were reconstructed by using PHYLIP (Felsenstein, 1993) (algorithm F84 as substitution model) and PHYML (algorithm general time-reversible, GTR, as substitution model plus gamma-distribution, plus invariant sites) (Guindon \& Gascuel, 2003), respectively. The robustness of the trees was evaluated by bootstrap analysis of 1000 resamplings for $\mathrm{NJ}$ and 100 resamplings for ML. The ML $16 \mathrm{~S}$ rRNA gene-based tree is shown in Fig. 1. The three isolates formed a robust phylogenetic clade supported by high bootstrap values and distinct from other genera in the family Veillonellaceae, in particular from the genus Dialister. These results were congruent with those observed from the NJ tree (data not shown). The DNA G $+\mathrm{C}$ content could not be determined, because the very limited growth of the strains in broth media meant that it was not possible to obtain enough biomass for $\mathrm{G}+\mathrm{C}$ content analysis. We used a genomic approach based on pulsedfield gel electrophoresis (PFGE) to study large-scale chromosome structure, which has been described as a sensitive indicator of phylogenetic relationships between bacteria of the same genus or species (Liu et al., 1999). This approach was previously applied to other members of the family Veillonellaceae and the rrn skeleton, studied after restriction of the DNA with the intron-encoded endonu- 
$\longmapsto .1$

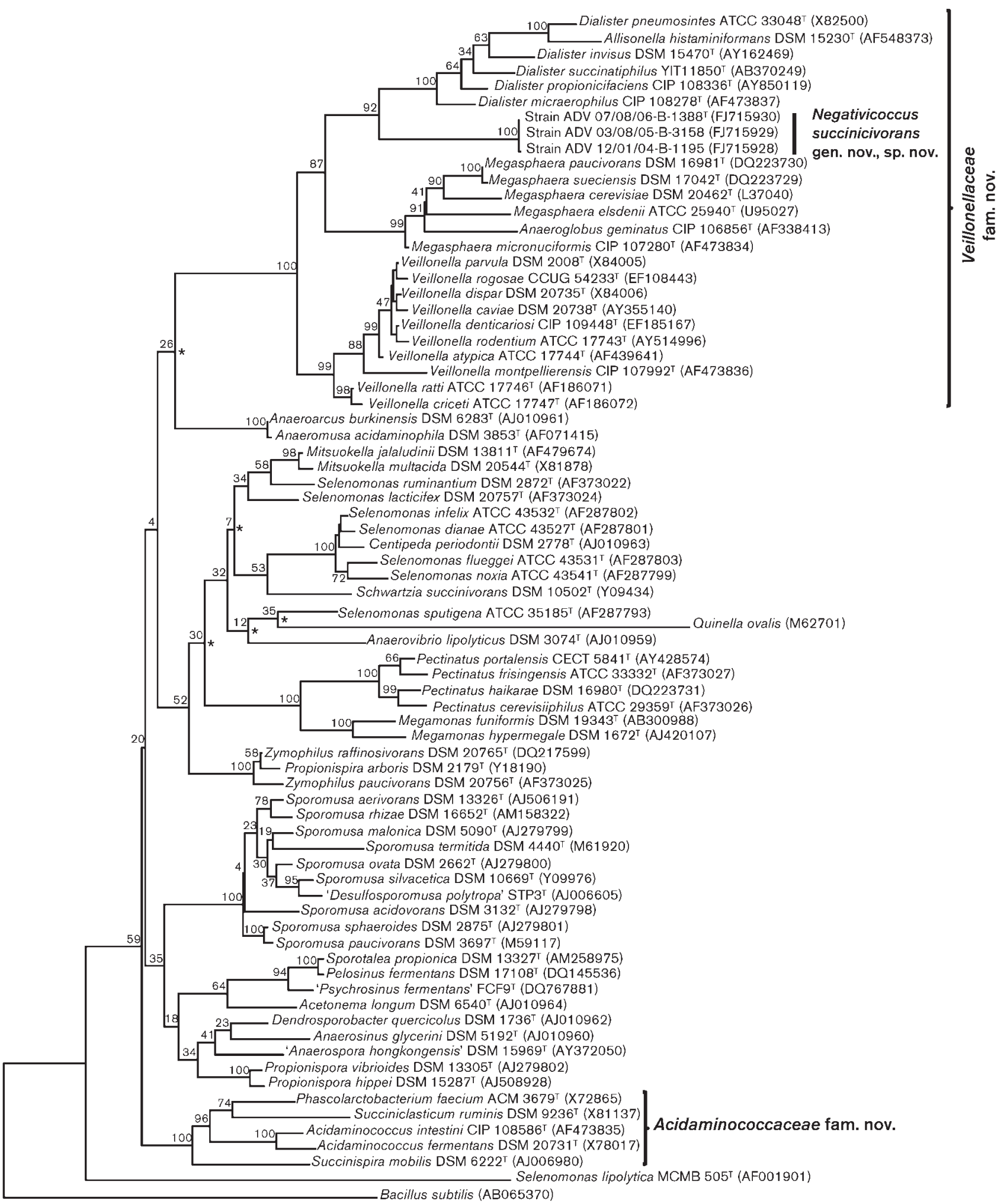

Fig. 1. ML tree based on partial $16 \mathrm{~S}$ rRNA gene sequences $(1211 \mathrm{nt})$, showing relationships between $N$. succinicivorans and other genera of the family Veillonellaceae. Bacillus subtilis was used as the outgroup. Numbers at nodes indicate percentage bootstrap support, based on analysis of 100 replicates. Nodes with asterisks varied according the method used to generate the tree. Bar, 0.1 substitutions per site. 
clease I-CeuI, appeared to be species-specific (Marchandin et al., 2003a; Jumas-Bilak et al., 2005, 2007). The number and sizes of bacterial chromosomes were analysed by PFGE of intact DNAs, and mapping experiments with I-CeuI (New England Biolabs) were undertaken as described previously (Marchandin et al., 2003a). The three strains studied possessed a unique chromosome with mean genomic size estimated to be $1.62 \mathrm{Mb}$. The chromosome migrated in PFGE as a faint band, suggesting a circular topology (Allardet-Servent et al., 1993). The three strains possessed four $r r n$ operons and displayed a common I-CeuI profile that differed from those of related species such as species of the genus Dialister (Jumas-Bilak et al., 2005). The large-scale chromosome structure of the three strains studied and relatives is available as Fig. S1 in IJSEM Online. The three isolates formed circular, convex, translucent and tiny colonies of less than $0.5 \mathrm{~mm}$ in diameter on Columbia sheep blood agar plates after $48 \mathrm{~h}$ incubation in an anaerobic jar with the Anaerogen System (Oxoid Unipath). The bacteria displayed a Gram-negative stain and were non-sporulating, non-motile and coccoid. Strains ADV 07/08/06-B-1388 ${ }^{\mathrm{T}}=$ AIP $149.07^{\mathrm{T}}$ and ADV $12 /$ 01/04-B-1195=AIP 150.07 were prepared for electron microscopy as described previously for both ultrathin sections observation and negative staining (Marchandin et al., 2003a; Jumas-Bilak et al., 2005). They presented as small cocci of about $0.4 \mu \mathrm{m}$ in diameter (Fig. 2A, B) and an outer membrane typical of Gram-negative surface layers was observed in ultrathin sections [Fig. $2 \mathrm{~B}(\mathrm{c})$ ], as described previously for several members of the family Veillonellaceae (Jumas-Bilak et al., 2004).

The strains were identified according to the procedures of the VPI Anaerobe Laboratory Manual (Holdeman et al., 1977). Susceptibility to special-potency discs was determined as described by Jousimies-Somer et al. (2002). Growth in microaerophilic conditions was tested using Campygen Compact (Oxoid Unipath). Metabolic end products were assayed by quantitative GC as described by Carlier (1985). For further biochemical characterization, the strains were grown anaerobically in trypticase/glucose/ yeast extract (TGY) broth and incubated at $37{ }^{\circ} \mathrm{C}$ for $72 \mathrm{~h}$ in an anaerobic jar containing $5 \% \mathrm{H}_{2}, 5 \% \mathrm{CO}_{2}$ and $90 \%$ $\mathrm{N}_{2}$ (by vol.). The strains were asaccharolytic; acid was not produced from fructose, glucose, lactose, maltose, mannose and sucrose. The isolates were non-reactive towards conventional biochemical tests (nitrate reduction, nitrite reduction, production of gas, catalase, indole and urease). They grew in microaerophilic conditions. The three strains displayed susceptibility to a bile disk ( $1 \mathrm{mg}$ ), and to

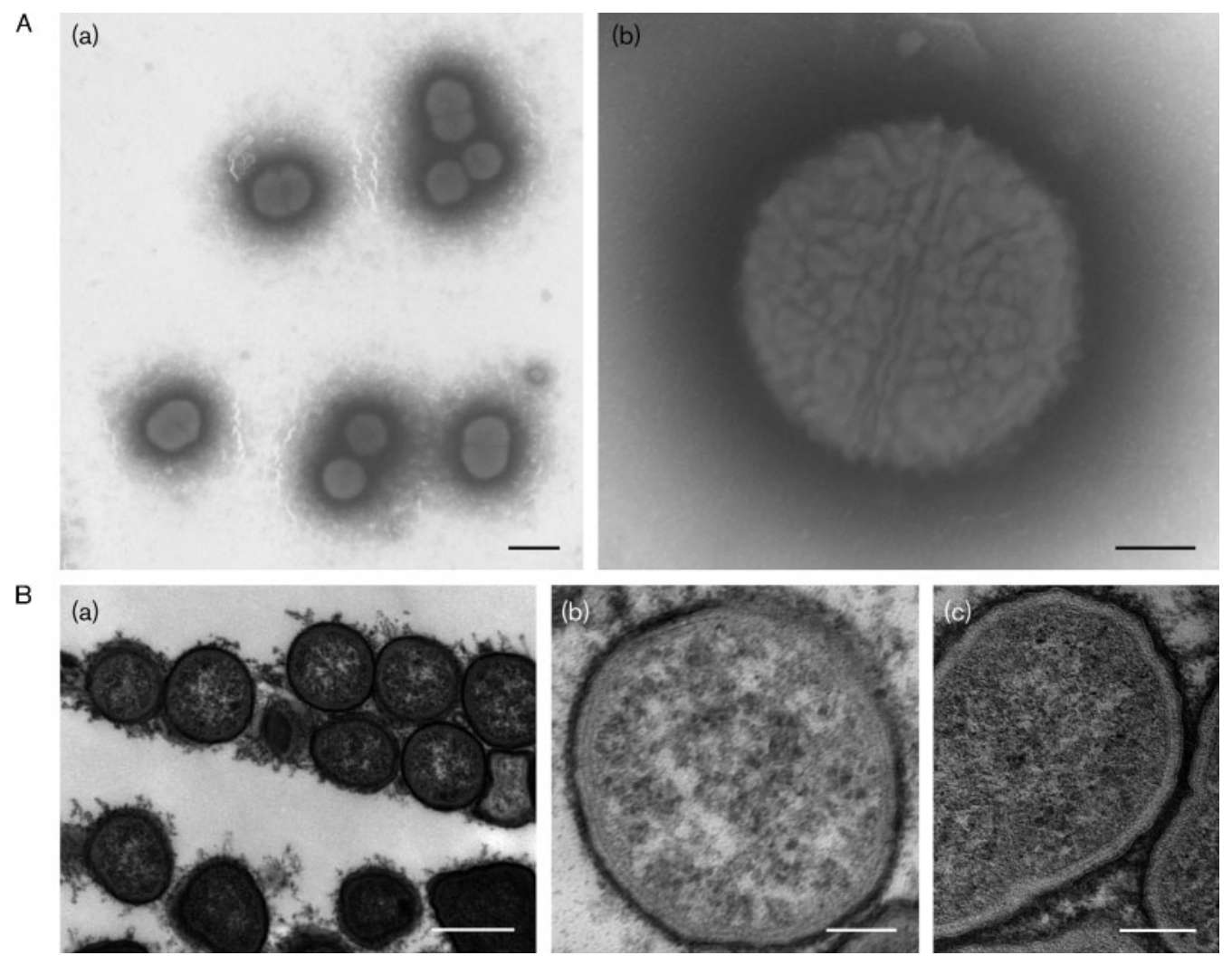

Fig. 2. (A) General morphology of $N$. succinicivorans strain ADV $07 / 08 / 06-B-1388^{\top}$ after negative staining. Bars, $500 \mathrm{~nm}(\mathrm{a})$ and $100 \mathrm{~nm}$ (b). (B) Electron microscopy of ultrathin sections to show ultrastructure of $N$. succinicivorans strain ADV 12/01/ 04-B-1195; ( $a, b)$ general morphology; (c) Gram-negative type cell wall with outer membrane. Bars: $500 \mathrm{~nm}(\mathrm{a}), 100 \mathrm{~nm}$ (b, c). 
kanamycin $(500 \mu \mathrm{g})$ and metronidazole $(50 \mu \mathrm{g})$ disks, and showed resistance to vancomycin $(5 \mu \mathrm{g})$ and colistin $(10 \mu \mathrm{g})$ disks (Rosco). Enzymic profiles obtained with Rapid ID 32 A (API, bioMérieux) showed arginine arylamidase activity for all three strains and alkaline phosphatase activity for strains ADV 12/01/04-B-1195= AIP 150.07 and ADV 03/08/05-B-3158=AIP 147.07. Major metabolic end products in TGY broth were acetic and propionic acids (2.2-2.4 and $1.2-1.5 \mathrm{mmol} \mathrm{l}^{-1}$, respectively). The strains also produced trace amounts (0.1$0.2 \mathrm{mmol} \mathrm{l}^{-1}$ ) of 2-hydroxyvaleric acid. In addition, strain ADV 12/01/04-B-1195=AIP 150.07 produced lactic acid $\left(2.4 \mathrm{mmol} \mathrm{l}^{-1}\right)$. Sodium succinate enhanced the growth of the three strains, whereas lactate and glutamate did not. Subsequent GC analysis revealed that the three strains produced a larger amount of propionate from TGY supplemented with sodium succinate (33.9-47.2 $\mathrm{mmol} \mathrm{l}^{-1}$ ), and a larger amount of 2-hydroxyvaleric acid (2.9-4.1 $\mathrm{mmol}^{-1}$ ) was also noted. Characteristics that differentiate the three strains from closely related taxa of the family Veillonellaceae are presented in Table 1.

Based on phenotypic, genotypic and phylogenetic evidence, a new genus, Negativicoccus gen. nov., is proposed for the three strains studied with one species, Negativicoccus succinicivorans sp. nov. Moreover, it is suggested that the uncultured bacterial clone rRNA030 and several uncultured clones from the human skin microbiome (Grice et al., 2009) probably belong to the species N. succinicivorans.

\section{Proposal to elevate the current family Veillonellaceae to class rank in the phylum Firmicutes, and description of Negativicutes classis nov.}

A 16S rRNA gene-based phylogeny was reconstructed using sequences of both cultured and non-cultured representative taxonomic units of different phyla selected according to their length (more than $1200 \mathrm{bp}$ ) and quality (fewer than $0.5 \%$ of undetermined positions) in the Greengenes database (http://greengenes.lbl.gov), and aligned using NAST program. Different datasets were used. Dataset A represented the phylum Firmicutes and included sequences of Veillonellaceae analysed in the tree in Fig. 1. The Firmicutes sequences were chosen on the basis of the diversity between the different taxonomic units belonging to this phylum according to Hugenholtz and Ludwig classifications in the Greengenes database. A total of 162 sequences were selected to represent the phylum Firmicutes sensu stricto, excluding Tenericutes. Among them, 62 were selected from uncultured clones and 63 were from type strains of the corresponding species. Dataset B included

Table 1. Characteristics differentiating the genus Negativicoccus from related genera of anaerobic Gram-negative bacteria

Data were taken from Rogosa (1984), Holt et al. (1994), Carlier et al. (2002), Marchandin et al. (2003a) and Jumas-Bilak et al. (2007). The phylogenetically related genus Allisonella was not included due to its unique phenotypic characteristics. Briefly, this ovoid-shaped, Gram-negative bacterium uses histidine decarboxylation as the sole source of energy since carbohydrates and organic acids are not used. Lysine can also be used but does not permit bacterial growth. The G $+\mathrm{C}$ content of the DNA is $46.8 \mathrm{~mol} \%$ (Garner et al., 2002). A, Acetic acid; P, propionic acid; L, lactic acid; B, butyric acid; V, valeric acid; C, caproic acid; iB, isobutyric acid; iV, isovaleric acid; S, succinic acid; ND, not detected.

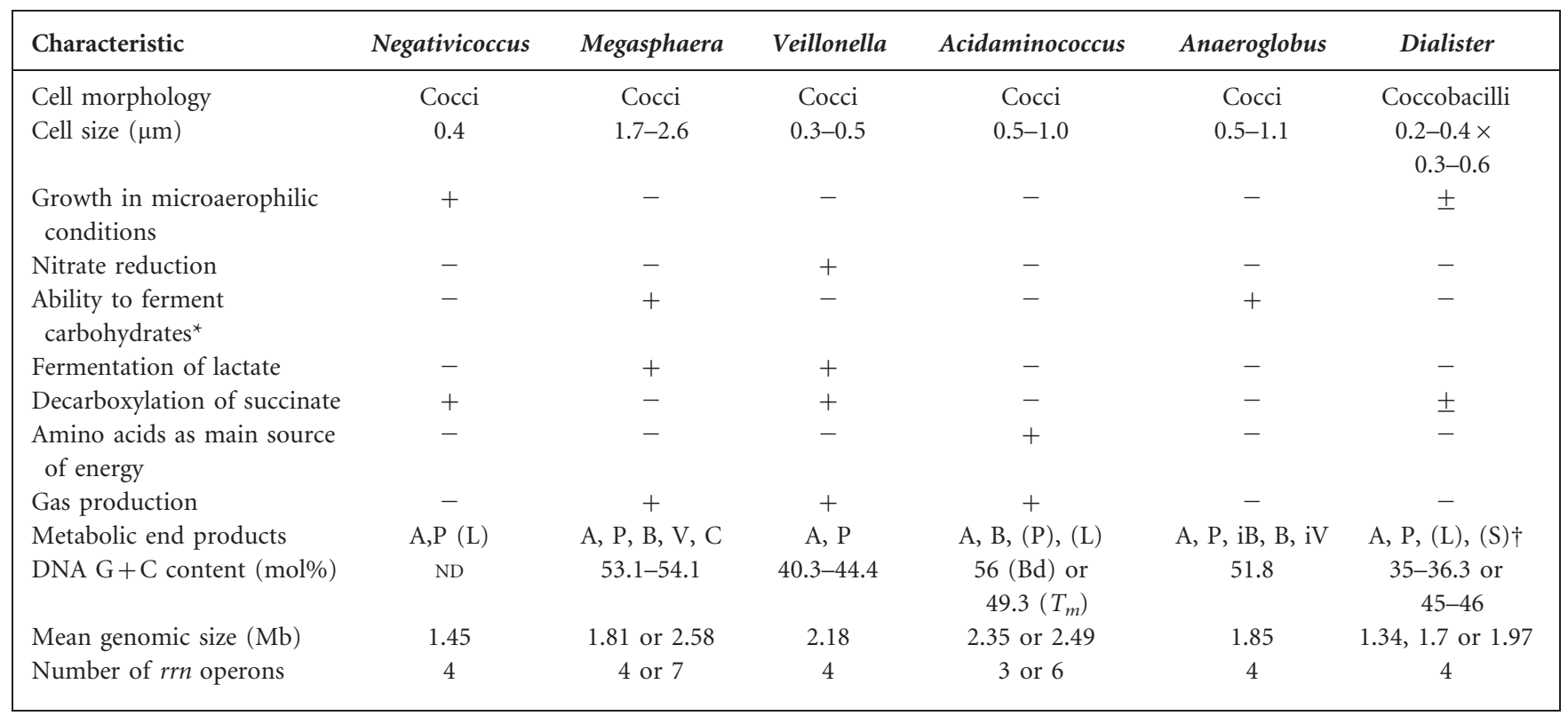

${ }^{*}$ Weak reactions observed for some species were not taken into account.

$\dagger$ Not detected in significant quantities for Dialister micraerophilus. 
sequences of the phyla Acidobacteria, Actinobacteria, Aquificae, Bacteroidetes and Chlamydiae. The composition of the two datasets is available as Table S1 in IJSEM Online. Datasets representative of other described bacterial phyla (Chlorobi, Chloroflexi, Chrysiogenetes, Cyanobacteria, Deferribacteres, Ferribacter, Fervidomicrobium, Fibrobacteres, Fusobacteria, Gemmanimonadetes, Haloanaerobacteriales, Lentisphaera, Moorella, Natroanaerobium, Nitrospirae, Planctomycetes, Poribacteria, Proteobacteria, Spirochaetae, Sulfobacilli, Synergistetes, Thermi, Thermacetogenium, Thermodesulfobacterium, Thermovernabulae and Verrumicrobia) corresponded to sequences selected in datasets 1, 2, 3, 4 and 5 used for the description of the phylum Synergistetes (Jumas-Bilak et al., 2009). Phylogenetic analyses were conducted by three methods, NJ, ML and Bayesian inference, as described previously (Jumas-Bilak et al., 2009). Phylogenetic trees were reconstructed from the datasets $\mathrm{A}+\mathrm{B}$ (Fig. S2 in IJSEM Online) and from datasets $\mathrm{A}+1$, $\mathrm{A}+2, \mathrm{~A}+3, \mathrm{~A}+4$ and $\mathrm{A}+5$ (data not shown). When compared with phylum-level taxa of each dataset, members of the family Veillonellaceae always aggregated to the phylum Firmicutes, whereas each described phylum formed an independent branch (Fig. S2 and data not shown). The same relationship between Veillonellaceae and Firmicutes or other phyla was observed whatever the phylogenetic method employed (data not shown). This provided strong evidence that on the basis of the 16S rRNA gene sequence Veillonellaceae belongs to the phylum Firmicutes.

However, members of the family Veillonellaceae are unified in a deep-branching clade, suggesting that Veillonellaceae is a taxon higher than family. The tree reconstructed with the datasets A + B (Fig. S2) showed that the clade corresponding to Veillonellaceae formed a robust lineage in the Firmicutes (bootstrap values of $99 \%$ and $927 \%$ in ML and NJ analyses, respectively, and posterior probability of 0.99 for Bayesian inference). Although very low significance of the relative order of branches connecting the Firmicutes classes was observed, the Veillonellaceae lineage was clearly separated from the classes 'Bacilli', Thermolithobacteria and 'Erysipelotrichi' as well as from the class 'Clostridia', the polyphyletic class where the family Veillonellaceae is currently classified. Therefore, class rank is proposed for the current family Veillonellaceae. Moreover, the class 'Clostridia' should probably be split into several taxa of various taxonomic levels and some of them should probably be elevated to a higher rank than that of class, as suggested by Ludwig et al. (2009). The new class is characterized by a major ultrastructural character, since all its members harbour a typical Gram-negative cell wall structure with an outer membrane as observed in electron microscopy (Bladen \& Mergenhagen, 1964; Kamio \& Takahashi, 1980; Kalmokoff et al., 2000; Males et al., 1984; Rogosa, 1969; Jumas-Bilak et al., 2007). With reference to this structure, we propose the name Negativicutes for the novel class.

In addition to phylogenetic evidence, the cell wall structure confirmed the separation of the proposed class Nega- tivicutes from other members of the phylum Firmicutes. In this class, a robust lineage consistently recovered from the diverse phylogenetic analyses, including diverse datasets and diverse methods (Fig. S2 and data not shown), groups all the members of the current family Veillonellaceae. This lineage, supported by high bootstrap values $(92 \%$ and $801 \%$ in ML and NJ analyses, respectively, and posterior probability of 0.98 for Bayesian inference), was described as the order Selenomonadales ord. nov. on the basis of the name of the first characterized genus in the taxon. Besides Selenomonadales ord. nov., a second robust lineage including six clones might well correspond to another order-level taxon in the class Negativicutes. The intra-order tree topology reveals the presence of two phylogenetically distinct and robust lineages, which are also consistently recovered from phylogenetic analyses. We propose the name Acidaminococcaceae fam. nov. for one of these clades (Fig. S2) supported by bootstrap values of $100 \%$ and $928 \%$ in ML and NJ analyses, respectively, and posterior probability of 1.00 for Bayesian inference. We also propose an emended description of the family Veillonellaceae for the second clade (Fig. S2) supported by bootstrap values of $100 \%$ and $1000 \%$ in ML and NJ analyses, respectively, and posterior probability of 0.93 for Bayesian inference.

Other branches at the subclass level could not be robustly aggregated and remain as Selenomonadales incertae sedis, but they may well represent several novel bacterial taxa. Further reappraisal will be warranted when their taxonomic position becomes more clearly delineated as new sequence data and/or data from analysis of other phylogenetic markers becomes available. The Grampositive/Gram-negative dichotomy appears as a structural character with evolutionary significance since it has been generally confirmed by molecular phylogeny based on $16 \mathrm{~S}$ rRNA sequences, in spite of some exceptions (Woese, 1987), but also on the basis of Hsp70 sequence signatures (Gupta, 1998). The major significance of the cell wall structure was also highlighted in the bacterial megaclassification proposed by Cavalier-Smith (2002). Gram-positive cell wall structure appears in only two eubacterial phyla, Firmicutes and Actinobacteria. The Firmicutes were proposed before the genetic era as a division in the kingdom Procaryotae (Gibbons \& Murray, 1978) along with the divisions Gracilicutes (Gram-negative bacteria) and Mollicutes (bacteria without peptidoglycan). The Firmicutes comprised three class-level taxa, including bacteria with different cell wall structure: 'Bacilli', 'Clostridia' and Mollicutes (Ludwig \& Klenk, 2001). A proposal to transfer the Mollicutes to the phylum Tenericutes on the basis of their unique phenotypic properties and the general low support by alternative markers (Ludwig \& Schleifer, 2005) led to the characterization of the class 'Erysipelotrichi' to accommodate wall-forming Gram-positive organisms of the family Erysipelotrichaceae previously classified with the Mollicutes in the phylum Firmicutes (Ludwig \& Schleifer, 2005). The classes 'Bacilli' and Thermolithobacteria include Gram-positive type organisms and thus the paraphyletic 
class 'Clostridia' is currently the only class in the phylum Firmicutes grouping both Gram-positive and Gram-negative taxa. The question of the belonging of Gram-negative members of the 'Clostridia' to the phylum Firmicutes remains open. Gram-negative members of the family Syntrophomonadaceae, class 'Clostridia', were recently reclassified in the phylum Synergistetes (Jumas-Bilak et al., 2009). On the contrary, we showed here that the novel class Negativicutes could not be excluded from the phylum Firmicutes on the basis of $16 \mathrm{~S}$ rRNA gene phylogeny. The proposed Gram-negative class Negativicutes is more closely related to Gram-positive bacteria of the phylum Firmicutes than to any other Gram-negative bacteria. Moreover, the lack of the diderm insert was observed in all representatives of the Negativicutes for which Hsp70 sequences are available in the GenBank database, and confirmed in this study in the proposed genus Negativicoccus. The inclusion in the phylum Firmicutes of other bacteria with a well-documented Gram-negative structure, such as Thermohalobacter (Cayol et al., 2000), Caloranaerobacter (Wery et al., 2001) and Caminicella (Alain et al., 2002), warrants further investigation for a better understanding of the evolutionary relationships between Gram-positive and Gram-negative bacteria. Currently classified in the family Clostridiaceae, probable reclassification of these genera has already been evoked by Ludwig et al. (2009), but has still to be studied.

\section{Description of Negativicoccus gen. nov.}

Negativicoccus [Ne.ga.ti.vi.coc'cus. L. adj. negativus negative; N.L. masc. n. coccus (from Gr. masc. n. kokkos) grain or berry; N.L. masc. n. Negativicoccus coccus with a typical Gram-negative cell wall structure with an outer membrane observed by electron microscopy].

Characteristics of the genus are as described below for the type and only species N. succinicivorans. Can be differentiated from other genera of the family Veillonellaceae by metabolic end products, and 16S rRNA gene and dnaK sequencing.

The type species is Negativicoccus succinicivorans.

\section{Description of Negativicoccus succinicivorans sp. nov.}

Negativicoccus succinicivorans (suc.ci.ni.ci.vo'rans. N.L. n. acidum succinicum succinic acid; L. part. adj. vorans devouring; N.L. part. adj. succinicivorans succinic aciddevouring).

Cells are coccoid $(0.4 \mu \mathrm{m}$ in diameter), Gram-negative, non-motile, non-sporulating. Colonies on Columbia blood agar are very tiny, less than $0.5 \mathrm{~mm}$ in diameter after $48 \mathrm{~h}$, circular, convex and translucent. Anaerobic and microaerophilic. Unreactive in most conventional biochemical tests. Asaccharolytic. Arginine arylamidase activity is noted and inconstant alkaline phosphatase activity is observed.
Metabolic end products are acetic and propionic acids and trace amounts of 2-hydroxyvaleric acid. Lactic acid may be produced. Growth is enhanced by sodium succinate.

The type strain is strain ADV 07/08/06-B- $1388^{\mathrm{T}}$ (=AIP $149.07^{\mathrm{T}}=$ CIP $109806^{\mathrm{T}}=$ DSM $21255^{\mathrm{T}}=$ CCUG $56017^{\mathrm{T}}$ ), and a reference strain is ADV 12/01/04-B-1195=AIP $150.07=$ CIP $109807=$ DSM $21256=$ CCUG 56016. Found in human clinical samples and the human skin microbiome.

\section{Emended description of the family Veillonellaceae Rogosa 1971}

The family Veillonellaceae includes the recognized genera Allisonella, Anaeroglobus, Dialister, Megasphaera, Veillonella and the proposed genus Negativicoccus.

The description is as described by Rogosa (1971) with the following modifications. Gram-negative bacteria. Cocci or coccobacilli. Anaerobic or microaerophilic. Gas may or may not be produced.

The type genus is Veillonella Prévot 1933.

\section{Description of the family Acidaminococcaceae}

Acidaminococcaceae (A.ci.dam.in.o.coc.ca'ce.ae. N.L. masc. n. Acidaminococcus type genus of the family; -aceae ending to denote a family; N.L. fem. pl. n. Acidaminococcaceae the Acidaminococcus family).

The family Acidaminococcaceae includes the recognized genera Acidaminococcus, Phascolarctobacterium, Succinispira and Succiniclasticum.

Gram-negative bacteria. Anaerobic. Non-sporulating. Cocci, or curved or pleiomorphic rods. Motile or not. Carbohydrates are not fermented (with the exception of some Acidaminococcus fermentans strains for which weak fermentation of glucose is observed). Growth with succinate or propionate production is observed or not. Amino acids are used as energy source or not.

The type genus is Acidaminococcus Rogosa 1969.

\section{Description of Selenomonadales ord. nov.}

Selenomonadales (Se.le.no.mo.na.da'les. N.L. fem. n. Selenomonas -adis type genus of the order as the first characterized genus; -ales ending to denote an order; N.L. fem. pl. n. Selenomonadales the Selenomonas order).

The description is the same as for the class Negativicutes. The order includes the validated genera Acetonema, Acidaminococcus, Allisonella, Anaeroarcus, Anaeroglobus, Anaeromusa, Anaerosinus, Anaerovibrio, Centipeda, Dendrosporobacter, Dialister, Megamonas, Megasphaera, Mitsuokella, Pectinatus, Pelosinus, Phascolarctobacterium, Propionispira, Propionispora, Quinella, Schwartzia, Selenomonas, Sporomusa, Sporotalea, Succiniclasticum, Succinispira, Thermosinus, Veillonella and Zymophilus, 
and the proposed genera Anaerospora, Desulfosporomusa, Psychrosinus and Negativicoccus.

The type genus is Selenomonas von Prowazek 1913.

\section{Description of Negativicutes classis nov.}

Negativicutes (Ne.ga.ti.vi.cu'tes. L. adj. negativus negative; L. fem. n. cutis skin; N.L. fem. pl. n. Negativicutes division with cells bounded by skin with two concentric lipid bilayers, the cytoplasmic membrane and an outer membrane, to indicate Gram-negative type of cell wall in the Firmicutes division).

The class Negativicutes is defined in phylogenetic terms on the basis of $16 \mathrm{~S}$ rRNA gene phylogeny. Members of the class harbour a typical Gram-negative cell wall structure with an outer membrane observable by electron microscopy.

Type order is Selenomonadales.

\section{Acknowledgements}

We thank I. Zorgniotti, R. Devine, S. Roux, M. Bedora-Faure, G. $\mathrm{K}$ 'ouas and M. Manich for excellent technical assistance. This work is partly supported by the association ADEREMPHA, Sauzet, France.

\section{References}

Alain, K., Pignet, P., Zbinden, M., Quillevere, M., Duchiron, F., Donval, J. P., Lesongeur, F., Raguenes, G., Crassous, P. \& other authors (2002). Caminicella sporogenes gen. nov., sp. nov., a novel thermophilic spore-forming bacterium isolated from an East-Pacific Rise hydrothermal vent. Int J Syst Evol Microbiol 52, 1621-1628.

Allardet-Servent, A., Michaux-Charachon, S., Jumas-Bilak, E., Karayan, L. \& Ramuz, M. (1993). Presence of one linear and one circular chromosome in the Agrobacterium tumefaciens C58 genome. $J$ Bacteriol 175, 7869-7874.

Altschul, S. F., Madden, T. L., Schäffer, A. A., Zhang, J., Zhang, Z., Miller, W. \& Lipman, D. J. (1997). Gapped BLAST and PSI-BLAST: a new generation of protein database search programs. Nucleic Acids Res 25, 3389-3402.

Bladen, H. A. \& Mergenhagen, S. E. (1964). Ultrastructures of Veillonella and morphological correlation of an outer membrane with particles associated with endotoxic activity. J Bacteriol 88, 14821492.

Carlier, J.-P. (1985). Gas chromatography of fermentation products: its application in diagnosis of anaerobic bacteria. Bull Inst Pasteur 83, 57-69.

Carlier, J.-P., Marchandin, H., Jumas-Bilak, E., Lorin, V., Henry, C., Carrière, C. \& Jean-Pierre, H. (2002). Anaeroglobus geminatus gen. nov., sp. nov., a novel member of the family Veillonellaceae. Int J Syst Evol Microbiol 52, 983-986.

Castresana, J. (2000). Selection of conserved blocks from multiple alignments for their use in phylogenetic analysis. Mol Biol Evol 17, 540-552.

Cavalier-Smith, T. (2002). The neomuran origin of archaebacteria, the negibacterial root of the universal tree and bacterial megaclassification. Int J Syst Evol Microbiol 52, 7-76.

Cayol, J. L., Ducerf, S., Patel, B. K., Garcia, J. L., Thomas, P. \& Ollivier, B. (2000). Thermohalobacter berrensis gen. nov., sp. nov., a thermophilic, strictly halophilic bacterium from a solar saltern. Int $J$ Syst Evol Microbiol 50, 559-564.

Felsenstein, J. (1993). PHYLIP (phylogeny inference package), version 3.5c. Distributed by the author. Department of Genome Sciences, University of Washington, Seattle, USA.

Garner, M. R., Flint, J. F. \& Russell, J. B. (2002). Allisonella histaminiformans gen. nov., sp. nov. A novel bacterium that produces histamine, utilizes histidine as its sole energy source, and could play a role in bovine and equine laminitis. Syst Appl Microbiol 25, 498506.

Garrity, G. M. \& Holt, J. G. (2001). Taxonomic outline of the Archaea and Bacteria. In Bergey's Manual of Systematic Bacteriology, 2nd edn, vol. 1, pp. 155-166. Edited by D. R. Boone \& R. W. Castenholz. New York: Springer.

Gibbons, N. E. \& Murray, R. G. E. (1978). Proposals concerning the higher taxa of bacteria. Int J Syst Bacteriol 28, 1-6.

Grice, E. A., Kong, H. H., Conlan, S., Deming, C. B., Davis, J., Young, A. C., NISC Comparative Sequencing Program, Bouffard, G. G., Blakesley, R. W. \& other authors (2009). Topographical and temporal diversity of the human skin microbiome. Science 324, 1190-1192.

Guindon, S. \& Gascuel, O. (2003). A simple, fast, and accurate algorithm to estimate large phylogenies by maximum likelihood. Syst Biol 52, 696-704.

Gupta, R. S. (1998). Protein phylogenies and signature sequences: a reappraisal of evolutionary relationships among archaebacteria, eubacteria, and eukaryotes. Microbiol Mol Biol Rev 62, 1435-1491.

Holdeman, L. V., Cato, E. P. \& Moore, W. E. C. (1977). Anaerobe Laboratory Manual, 4th edn. Blacksburg, VA: Virginia Polytechnic Institute and State University.

Holt, J. G., Krieg, N. R., Sneath, P. H. A., Staley, J. T. \& Williams, S. T. (1994). Anaerobic Gram-negative cocci. In Bergey's Manual of Determinative Bacteriology, 9th edn, pp. 347-350. Edited by J. G. Holt. Baltimore: Lippincott Williams \& Wilkins.

Jousimies-Somer, H. R., Summanen, P., Citron, D. M., Baron, E. J., Wexler, H. M. \& Finegold, S. M. (2002). Wadworth-KTL Anaerobic Bacteriology Manual, 6th edn. Belmont, CA, USA: Star Publishing Company.

Jumas-Bilak, E., Carlier, J.-P., Jean-Pierre, H., Teyssier, C., Gay, B., Campos, J. \& Marchandin, H. (2004). Veillonella montpellierensis sp. nov., a novel, anaerobic, Gram-negative coccus isolated from human clinical samples. Int J Syst Evol Microbiol 54, 1311-1316.

Jumas-Bilak, E., Jean-Pierre, H., Carlier, J.-P., Teyssier, C., Bernard, K., Gay, B., Campos, J., Morio, F. \& Marchandin, H. (2005). Dialister micraerophilus sp. nov. and Dialister propionicifaciens sp. nov., isolated from human clinical samples. Int J Syst Evol Microbiol 55, 2471-2478.

Jumas-Bilak, E., Carlier, J.-P., Jean-Pierre, H., Mory, F., Teyssier, C., Gay, B., Campos, J. \& Marchandin, H. (2007). Acidaminococcus intestini sp. nov., isolated from human clinical samples. Int J Syst Evol Microbiol 57, 2314-2319.

Jumas-Bilak, E., Roudiere, L. \& Marchandin, H. (2009). Description of Synergistetes phyl. nov. and emended description of the phylum Deferribacteres and of the family Syntrophomonadaceae, phylum Firmicutes. Int J Syst Evol Microbiol 59, 1028-1035.

Kalmokoff, M. L., Austin, J. W., Whitford, M. F. \& Teather, R. M. (2000). Characterization of a major envelope protein from the rumen anaerobe Selenomonas ruminantium OB268. Can J Microbiol 46, 295 303.

Kamio, Y. \& Takahashi, H. (1980). Outer membrane proteins and cell surface structure of Selenomonas ruminantium. J Bacteriol 141, 899907. 
Liu, S.-L., Schryvers, A. B., Sanderson, K. E. \& Johnston, R. N. (1999). Bacterial phylogenetic clusters revealed by genome structure. J Bacteriol 181, 6747-6755.

Ludwig, W. \& Klenk, H.-P. (2001). Overview: a phylogenetic backbone and taxonomic framework for procaryotic systematics. In Bergey's Manual of Systematic Bacteriology, 2nd edn, vol. 1, pp. 49-65. Edited by D. R. Boone, R. W. Castenholz \& G. M. Garrity. New York: Springer.

Ludwig, W. \& Schleifer, K. H. (2005). Molecular phylogeny of bacteria based on comparative sequence analysis of conserved genes. In Microbial Phylogeny and Evolution, Concepts and Controversies, pp. 70-98. Edited by J. Sapp. New York: Oxford University Press.

Ludwig, W., Schleifer, K.-H. \& Whitman, W. B. (2009). Revised road map to the phylum Firmicutes. In Bergey's Manual of Systematic Bacteriology, 2nd edn, vol. 3 (The Firmicutes), pp. 1-13. Edited by P. De Vos, G. Garrity, D. Jones, N. R. Krieg, W. Ludwig, F. A. Rainey, K.-H. Schleifer \& W. B. Whitman. New York: Springer.

Males, B. M., Berthold, P., Dougherty, P. A. \& Listgarten, M. A. (1984). Helical flagellation in Centipeda periodontii, a Gram-negative, anaerobic bacillus from periodontitis lesions. J Gen Microbiol 130, 185-191.

Marchandin, H., Jumas-Bilak, E., Gay, B., Teyssier, C., Jean-Pierre, H., Siméon de Buochberg, M., Carrière, C. \& Carlier, J.-P. (2003a). Phylogenetic analysis of some Sporomusa sub-branch members isolated from human clinical specimens: description of Megasphaera micronuciformis sp. nov. Int J Syst Evol Microbiol 53, 547-553.

Marchandin, H., Teyssier, C., Siméon de Buochberg, M., JeanPierre, H., Carrière, C. \& Jumas-Bilak, E. (2003b). Intra-chromosomal heterogeneity between the four 16S rRNA gene copies in the genus Veillonella: implications for phylogeny and taxonomy. Microbiology 149, 1493-1501.
Prévot, A. R. (1933). Etude de systématique bactérienne. I. Lois générales. II. Cocci anaérobius. Ann Sci Nat Zool Biol Anim 15, $23-$ 260.

Rogosa, M. (1969). Acidaminococcus gen. n., Acidaminococcus fermentans sp. n., anaerobic Gram-negative diplococci using amino acids as the sole energy source for growth. J Bacteriol 98, 756766.

Rogosa, M. (1971). Transfer of Veillonella Prévot and Acidaminococcus Rogosa from Neisseriaceae to Veillonellaceae fam. nov. and the inclusion of Megasphaera Rogosa in Veillonellaceae. Int $J$ Syst Bacteriol 21, 231-233.

Rogosa, M. (1984). Anaerobic Gram-negative cocci. In Bergey's Manual of Systematic Bacteriology, vol. 1, pp. 680-685. Edited by N. R. Krieg \& J. G. Holt. Baltimore: Williams \& Wilkins.

Sokolova, T., Hanel, J., Onyenwoke, R. U., Reysenbach, A. L., Banta, A., Geyer, R., Gonzalez, J. M., Whitman, W. B. \& Wiegel, J. (2007). Novel chemolithotrophic, thermophilic, anaerobic bacteria Thermolithobacter ferrireducens gen. nov., sp. nov. and Thermolithobacter carboxydivorans sp. nov. Extremophiles 11, 145-157.

Wery, N., Moricet, J. M., Cueff, V., Jean, J., Pignet, P., Lesongeur, F., Cambon-Bonavita, M. A. \& Barbier, G. (2001). Caloranaerobacter azorensis gen. nov., sp. nov., an anaerobic thermophilic bacterium isolated from a deep-sea hydrothermal vent. Int J Syst Evol Microbiol 51, 1789-1796.

Willems, A. \& Collins, M. D. (1995). Phylogenetic placement of Dialister pneumosintes (formerly Bacteroides pneumosintes) within the Sporomusa subbranch of the Clostridium subphylum of the Grampositive bacteria. Int J Syst Bacteriol 45, 403-405.

Woese, C. R. (1987). Bacterial evolution. Microbiol Rev 51, 221-271. 